Vietnam Journal of Mechanics, VAST, Vol. 27, No. 2 (2006), pp. 111-119

\title{
INFLUENCE OF THE HARDENING CHARACTERISTICS OF MATERIAL ON THE CRITICAL LOAD IN THE ELASTO - PLASTIC STABILITY PROBLEM OF CONICAL SHELLS
}

\author{
DaO Huy BICH ${ }^{1}$ AND Vu KHAC BAY ${ }^{2}$ \\ 1 Vietnam National University, Hanoi \\ 2 University of Forestry
}

\begin{abstract}
In this paper by using the theory of elasto-plastic processes and adjacentequilibrium criterion the governing equations of the elasto-plastic stability problem of conical shells are derived. The Bubnov-Galerkin's method combined with the loading parameter method are applied in solving the mentioned problem. The influence of the hardening characteristics of material on the critical load is investigated.
\end{abstract}

\section{INTRODUCTION}

Analysis of the elasto-plastic stability problem of shells with homogeneous membrane stress of the prebuckling state was considered by some authors $[1 \div 4]$, but in the case when the prebuckling state is non- homogeneous many difficulties arise in solving the problem, because now stability equations are a set of partial differential equations with variable coefficients. Otherwise we can get more difficulties in determination of material functions occured in the constitutive relations, for example the secant modulus and tangent modulus which become functions of point coordinates. Furthermore in the shell occur elastic and plastic zones, the boundary of which is unknown, it must be determined simultaneously in the solution process.

In this paper the governing equations of the elasto-plastic stability problem of conical shells are developed based on the theory of elasto-plastic processes and the adjacentequilibrium criterion. The Bubnov-Galerkin's method combined with the loading parameter method can be applied in solving considered problem. A piece-wise linearization procedure of the material function figured in constitutive relations is demonstrated for material with general hardening characteristics and the influence of this characteristics on the critical load is investigated.

\section{PREBUCKLING STATE OF A CONICAL SHELL.}

Points in the middle surface of a conical shell may be referred to coordinates $(x, \theta)$, where $x$ is a coordinate taken from the shell top to the considered point in the generatrix direction, $\theta$ - a circumferential coordinate, $\alpha$ - an open angle at the shell top, $l$ - the length of shell anh $h$ - the shell thichness. The stress occurs in the prebuckling state depending on loading process, here we restrict ourselves the applied load is axisymmetric and the linear bending equations are used for the prebuckling.deformation. If the shell is acted on by external pressure with intensity $p$, the prebuckling stress state is of the form

$$
\sigma_{x}^{0}=-\frac{p x}{2 h} \operatorname{tg} \alpha, \quad \sigma_{\theta}^{0}=-\frac{p x}{h} \operatorname{tg} \alpha, \quad \sigma_{x \theta}^{0}=0, \quad \sigma_{u}^{0}=\frac{\sqrt{3} p x}{2 h} \operatorname{tg} \alpha
$$




$$
N_{x}^{0}=-\frac{p x}{2} \operatorname{tg} \alpha, \quad N_{\theta}^{0}=-p x \operatorname{tg} \alpha, \quad N_{x \theta}^{0}=0 .
$$

It is clear that along the shell generatrix the stress intensity increases linearly with respect to $x$. Thus at points $0 \leqslant x \leqslant x_{s}$ elastic state occurs, while $x_{s} \leqslant x \leqslant l$ the shell is in plastic state, the boundary of two zones is determined by

$$
x_{s}=\frac{2 \sigma_{s} h}{\sqrt{3} p \operatorname{tg} \alpha},
$$

where $\sigma_{s^{-}}$yield stress (when $\sigma_{u}^{0}=\sigma_{s}$ ), $x_{s^{-}}$elastic- plastic zones boundary. This boundary will be determined simultaneously in the solving process. Putting

$$
t=\frac{x}{l}, \quad \sigma_{u}^{0}=\frac{\sqrt{3} p l}{2 h} \operatorname{tg} \alpha \frac{x}{l}=\sigma_{M}^{0} t,
$$

where

$$
\sigma_{M}^{0}=\frac{\sqrt{3} p l}{2 h} \operatorname{tg} \alpha
$$

one can get

$$
0 \leqslant t \leqslant 1, \quad \sigma_{s}=\sigma_{M}^{0} t_{s}, \quad t_{s}=\frac{x_{s}}{l}=\frac{\sigma_{s}}{\sigma_{M}^{0}} .
$$

\section{STABILITY EQUATIONS}

Applying stability equations [5] for conical shells subjected to external pressure yields

$$
\begin{gathered}
R_{2} \rightarrow \infty, \quad \lim _{R_{2} \rightarrow \infty} R_{2} d \varphi=d x, \quad r=x \sin \alpha \\
\varphi=\pi / 2-\alpha, \quad \sin \varphi=\cos \alpha, \quad \cos \varphi=\sin \alpha \\
\frac{\partial}{\partial x}\left(x \delta N_{x}\right)+\frac{1}{\sin \alpha} \frac{\partial \delta N_{x \theta}}{\partial \theta}-\delta N_{\theta}=0, \\
\frac{1}{\sin \alpha} \frac{\partial \delta N_{\theta}}{\partial \theta}+\frac{1}{x} \frac{\partial}{\partial x}\left(x^{2} \delta N_{x \theta}\right)=0, \\
\frac{\partial^{2}}{\partial x^{2}}\left(x \delta M_{x}\right)+\frac{2}{\sin \alpha}\left(\frac{\partial^{2} \delta M_{x} \theta}{\partial x \partial \theta}+\frac{1}{x} \frac{\partial \delta M_{x \theta}}{\partial \theta}\right)+\frac{1}{x \sin ^{2} \alpha} \frac{\partial^{2} \delta M_{\theta}}{\partial \theta^{2}}- \\
-\frac{\partial \delta M_{\theta}}{\partial x}-\delta N_{\theta} \operatorname{cotg} \alpha+p \operatorname{tg} \alpha\left(x \frac{\partial \delta w}{\partial x}+\frac{x^{2}}{2} \frac{\partial^{2} \delta w}{\partial x^{2}}+\frac{1}{\sin ^{2} \alpha} \frac{\partial^{2} \delta w}{\partial \theta^{2}}\right)=0,
\end{gathered}
$$

with the following boundary conditions:

- at the fixed point 0 of the shell top

$$
\delta u=\delta v=\delta w=0 \quad \text { with } \quad x=0 ;
$$

- the end cross section of the shell is simply supported such that

$$
\delta w \quad=0, \quad \delta v=0, \quad \delta M_{x}=0, \quad \delta N_{x}=0 .
$$


Remark:

- With $\alpha=\pi / 2$ equations (3.1) reduce to stability equations for a circular plate.

- With $x \sin \alpha=a=$ const and $\alpha=0$, equations (3.1) become stability equations for a circular cylindrical shell of radius $a$.

According to the elasto-plastic process theory [3] the expressions for internal forces increments and internal moments increments of a conical shell subjected to external pressure are obtained as follows

$$
\begin{aligned}
& \delta N_{x}=h\left[\left(N+\frac{\Phi^{\prime}}{3}\right) \frac{\partial \delta u}{\partial x}+\frac{2}{3} \Phi^{\prime}\left(\frac{1}{x \sin \alpha} \frac{\partial \delta v}{\partial \theta}+\frac{\delta u}{x}+\frac{\delta w}{x} \operatorname{cotg} \alpha\right)\right], \\
& \delta N_{\theta}=h \frac{2}{3} \Phi^{\prime}\left[\frac{\partial \delta u}{\partial x}+2\left(\frac{1}{x \sin \alpha} \frac{\partial \delta v}{\partial \theta}+\frac{\delta u}{x}+\frac{\delta w}{x} \operatorname{cotg} \alpha\right)\right], \\
& \delta N_{x \theta}=h \frac{N}{3}\left[\frac{\partial \delta v}{\partial x}-\frac{\delta v}{x}+\frac{1}{x \sin \alpha} \frac{\partial \delta u}{\partial \theta}\right], \\
& \delta M_{x}=-\frac{h^{3}}{12}\left[\left(N+\frac{\Phi^{\prime}}{3}\right) \frac{\partial^{2} \delta w}{\partial x^{2}}+\frac{2}{3} \Phi^{\prime}\left(\frac{1}{x^{2} \sin ^{2} \alpha} \frac{\partial^{2} \delta w}{\partial \theta^{2}}+\frac{1}{3} \frac{\partial \delta w}{\partial x}\right)\right], \\
& \delta M_{\theta}=-\frac{h^{3}}{18} \Phi^{\prime}\left[\frac{\partial^{2} \delta w}{\partial x^{2}}+2\left(\frac{1}{x^{2} \sin ^{2} \alpha} \frac{\partial^{2} \delta w}{\partial \theta^{2}}+\frac{1}{x} \frac{\partial \delta w}{\partial x}\right)\right], \\
& \delta M_{x \theta}=-\frac{h^{3}}{18} N\left(\frac{1}{x \sin \alpha} \frac{\partial^{2} \delta w}{\partial x \partial \theta}-\frac{1}{x^{2} \sin \alpha} \frac{\partial \delta w}{\partial \theta}\right),
\end{aligned}
$$

where $N=\sigma_{u}^{0} / s=\Phi(s) / s$ plays a role of a secant modulus and $\Phi^{\prime}(s)$ - tangent modulus of the hardening material. In this case $N$ and $\Phi^{\prime}$ are functions of $x$ alone, $\delta u, \delta v, \delta w$ are displacement increments in generatrix, circumferential and normal to the shell directions, respectively. Since then without mistakes we can omit the word " $\delta$ " in the denotation of increment quantities.

Substituting (3.4) into (3.1) leads to equations

$$
\begin{aligned}
& x\left(3 N+\Phi^{\prime}\right) \frac{\partial^{2} u}{\partial x^{2}}+\left(3 N+\Phi^{\prime}+3 x \frac{\partial N}{\partial x}+x \frac{\partial \Phi^{\prime}}{\partial x}\right) \frac{\partial u}{\partial x}+\left(2 x \frac{\partial \Phi^{\prime}}{\partial x}-4 \Phi^{\prime}\right) \frac{u}{x}+ \\
& +\frac{N}{x \sin ^{2} \alpha} \frac{\partial^{2} u}{\partial \theta^{2}}+\left(N+2 \Phi^{\prime}\right) \frac{1}{\sin \alpha} \frac{\partial^{2} v}{\partial x \partial \theta}-\left(N+4 \Phi^{\prime}-2 x \frac{\partial \Phi^{\prime}}{\partial x}\right) \frac{1}{\sin \alpha} \frac{\partial v}{\partial \theta}+ \\
& +2 \Phi^{\prime} \operatorname{cotg} \alpha \frac{\partial w}{\partial x}-\left(4 \Phi^{\prime}-2 x \frac{\partial \Phi^{\prime}}{\partial x}\right) \operatorname{cotg} \alpha \frac{w}{x}=0 ; \\
& x N \frac{\partial^{2} v}{\partial x^{2}}+\left(N+x \frac{\partial N}{\partial x}\right) \frac{\partial v}{\partial x}-\left(N+x \frac{\partial N}{\partial x}\right) \frac{v}{x}+4 \frac{\Phi^{\prime}}{x \sin ^{2} \alpha} \frac{\partial^{2} v}{\partial \theta^{2}}+\left(N+2 \Phi^{\prime}\right) \frac{1}{\sin \alpha} \frac{\partial^{2} u}{\partial x \partial \theta}+ \\
& +\left(N+4 \Phi^{\prime}+x \frac{\partial N}{\partial x}\right) \frac{1}{x \sin \alpha} \frac{\partial u}{\partial \theta}+4 \frac{\Phi^{\prime}}{x \sin \alpha} \operatorname{cotg} \alpha \frac{\partial w}{\partial \theta}=0 ; \\
& x\left(3 N+\Phi^{\prime}\right) \frac{\partial^{4} w}{\partial x^{4}}+\left(6 N+2 \Phi^{\prime}+6 x \frac{\partial N}{\partial x}+2 x \frac{\partial \Phi^{\prime}}{\partial x}\right) \frac{\partial^{3} w}{\partial x^{3}}+ \\
& +\left(3 x \frac{\partial^{2} N}{\partial x^{2}}+6 \frac{\partial N}{\partial x}-4 \frac{\Phi^{\prime}}{x}+4 \frac{\partial \Phi^{\prime}}{\partial x}+x \frac{\partial^{2} \Phi^{\prime}}{\partial x^{2}}\right) \frac{\partial^{2} w}{\partial x^{2}}+\left(\frac{4}{x^{2}} \Phi^{\prime}-\frac{4}{x} \frac{\partial \Phi^{\prime}}{\partial x}+2 \frac{\partial^{2} \Phi^{\prime}}{\partial x^{2}}\right) \frac{\partial w}{\partial x}+
\end{aligned}
$$




$$
\begin{aligned}
& +4\left(N+\Phi^{\prime}\right) \frac{1}{x \sin ^{2} \alpha} \frac{\partial^{4} w}{\partial x^{2} \partial \theta^{2}}-4\left(N+\Phi^{\prime}-x \frac{\partial N}{\partial x}-x \frac{\partial \Phi^{\prime}}{\partial x}\right) \frac{1}{x^{2} \sin ^{2} \alpha} \frac{\partial^{3} w}{\partial x \partial \theta^{2}}+ \\
& +4\left(N+3 \Phi^{\prime}-x \frac{\partial N}{\partial x}-2 x \frac{\partial \Phi^{\prime}}{\partial x}+\frac{x^{2}}{2} \frac{\partial^{2} \Phi^{\prime}}{\partial x^{2}}\right) \frac{1}{x^{3} \sin ^{2} \alpha} \frac{\partial^{2} w}{\partial \theta^{2}}+4 \frac{\Phi^{\prime}}{x^{3} \sin ^{4} \alpha} \frac{\partial^{4} w}{\partial \theta^{4}}+ \\
& +\frac{48 \Phi^{\prime}}{h^{2}} \operatorname{cotg} \alpha\left(\frac{1}{2} \frac{\partial u}{\partial x}+\frac{1}{x \sin \alpha} \frac{\partial v}{\partial \theta}+\frac{u}{x}+\frac{w}{x} \operatorname{cotg} \alpha\right)+ \\
& -\frac{36 p}{h^{3}} \operatorname{tg} \alpha\left(x \frac{\partial w}{\partial x}+\frac{x^{2}}{2} \frac{\partial^{2} w}{\partial x^{2}}+\frac{1}{\sin ^{2} \alpha} \frac{\partial^{2} w}{\partial \theta^{2}}\right)=0 .
\end{aligned}
$$

\section{SOLVING METHOD}

a) In the case of elastic conical shells

$$
N=\Phi^{\prime}=3 G,
$$

equations (3.5) reduce to ones of elastic stability equations considered in [7].

b) In the case of conical shells made of material with linear hardening characteristics

$$
\Phi^{\prime}(s)=g=\mathrm{const}, \quad N=\frac{\sigma_{u}^{0}}{s}=\frac{g \sigma_{u}^{0}}{\sigma_{u}^{0}-\left(\sigma_{s}-g \varepsilon_{s}\right)}=g+\frac{g b}{\sigma_{u}^{0}-b},
$$

where $b=\sigma_{s}-g \varepsilon_{s}$, the set of equations (3.5) can be written as

$$
\begin{aligned}
& x\left(3 N+\Phi^{\prime}\right) \frac{\partial^{2} u}{\partial x^{2}}+\left(3 N+\Phi^{\prime}+3 x \frac{\partial N}{\partial x}\right) \frac{\partial u}{\partial x}-4 \Phi^{\prime} \frac{u}{x}+\frac{N}{x \sin ^{2} \alpha} \frac{\partial^{2} u}{\partial \theta^{2}}+ \\
& +\left(N+2 \Phi^{\prime}\right) \frac{1}{\sin \alpha} \frac{\partial^{2} v}{\partial x \partial \theta}-\left(N+4 \Phi^{\prime}\right) \frac{1}{\sin \alpha} \frac{\partial v}{\partial \theta}+2 \Phi^{\prime} \operatorname{cotg} \alpha \frac{\partial w}{\partial x}-4 \Phi^{\prime} \operatorname{cotg} \alpha \frac{w}{x}=0 \\
& x N \frac{\partial^{2} v}{\partial x^{2}}+\left(N+x \frac{\partial N}{\partial x}\right)\left(\frac{\partial v}{\partial x}-\frac{v}{x}\right)+4 \Phi^{\prime} \frac{1}{x \sin ^{2} \alpha} \frac{\partial^{2} v}{\partial \theta^{2}}+\left(N+2 \Phi^{\prime}\right) \frac{1}{\sin \alpha} \frac{\partial^{2} u}{\partial x \partial \theta}+ \\
& +\left(N+x \frac{\partial N}{\partial x}+4 \Phi^{\prime}\right) \frac{1}{x \sin \alpha} \frac{\partial u}{\partial \theta}+4 \Phi^{\prime} \frac{1}{x \sin \alpha} \operatorname{cotg} \alpha \frac{\partial w}{\partial \theta}=0 \\
& x\left(3 N+\Phi^{\prime}\right) \frac{\partial^{4} w}{\partial x^{4}}+\left(6 N+2 \Phi^{\prime}+6 x \frac{\partial N}{\partial x}\right) \frac{\partial^{3} w}{\partial x^{3}}+\left(3 x \frac{\partial^{2} N}{\partial x^{2}}+6 \frac{\partial N}{\partial x}-4 \frac{\Phi^{\prime}}{x}\right) \frac{\partial^{2} w}{\partial x^{2}}+ \\
& +4 \frac{\Phi^{\prime}}{x^{2}} \frac{\partial w}{\partial x}-4\left(N+\Phi^{\prime}-x \frac{\partial N}{\partial x}\right) \frac{1}{x^{2} \sin ^{2} \alpha} \frac{\partial^{3} w}{\partial x \partial \theta^{2}}+4\left(N+\Phi^{\prime}\right) \frac{1}{x \sin ^{2} \alpha} \frac{\partial^{4} w}{\partial x^{2} \partial \theta^{2}}+ \\
& +4\left(N+3 \Phi^{\prime}-x \frac{\partial N}{\partial x}\right) \frac{1}{x^{3} \sin ^{2} \alpha} \frac{\partial^{2} w}{\partial \theta^{2}}+4 \Phi^{\prime} \frac{1}{x^{3} \sin ^{4} \alpha} \frac{\partial^{4} w}{\partial \theta^{4}}+ \\
& +\frac{48 \Phi^{\prime}}{h^{2}} \operatorname{cotg} \alpha\left(\frac{1}{2} \frac{\partial u}{\partial x}+\frac{1}{x \sin ^{2}} \frac{\partial v}{\partial \theta}+\frac{u}{x}+\frac{w}{x} \operatorname{cotg} \alpha\right)+ \\
& -\frac{36 p}{h^{3}} \operatorname{tg} \alpha\left(x \frac{\partial w}{\partial x}+\frac{x^{2}}{2} \frac{\partial^{2} w}{\partial x^{2}}+\frac{1}{\sin ^{2} \alpha} \frac{\partial^{2} w}{\partial \theta^{2}}\right)=0 .
\end{aligned}
$$

By using these equations the stability problem has been investigated in [6]. Later equations (4.1) will be used as basic equations for solving step - by -step the stability problem of conical shell made of material with general hardening characteristics. 
c) In the case of conical shells made of general hardening material, relation $\sigma_{u}^{0}=\Phi(s)$ represents a material function precisely determined for a given material, it is a curve of the stress intensity $\sigma_{u}^{0}$ versus the arc - length $s$ of strain trajectory. In general the slope of a tangent to the material curve varies from point to point. Otherwise in the considered conical shell subjected to external pressure

$$
\sigma_{u}^{0}=\frac{\sqrt{3} p x}{2 h} \operatorname{tg} \alpha=\sigma_{M}^{0} t
$$

i.e. at each point on the generatrix we can know the value of stress intensity $\sigma_{u}^{0}$, which is respect to one point on the material curve with coordinates $\left(\sigma_{u}^{0}, s\right)$. Therefore within $s$ and $t$ there is a relation one - to - one, it means that with each value $t$ we can get the value $\sigma_{u}^{0}$, then according to the graph $\sigma_{u}^{0}=\Phi(s)$ we determine the value $s$ respectively and then the value $\Phi^{\prime}(s)$ (the slope of tangent to the material curve) and $N=\sigma_{u}^{0} / s$.

Implementing a linearization of the general hardening curve as a piecewise linear hardening one, such that in each interval $\left[s_{i}, s_{i+1}\right]$ we obtain $\Phi^{\prime}(s)=\Phi^{\prime}\left(s_{i}\right)=g_{i}=$ const. Consequently at different intervals the quantity $\Phi^{\prime}(s)$ gets different but constant values, then:

$$
\begin{gathered}
\frac{\partial \Phi^{\prime}}{\partial x}=\frac{\partial^{2} \Phi^{\prime}}{\partial x^{2}}=0 \quad \text { or } \quad \frac{\partial \Phi^{\prime}}{\partial t}=\frac{\partial^{2} \Phi^{\prime}}{\partial t^{2}}=0 \\
N=g_{i}+\bar{N} \quad \text { with } \quad \bar{N}=\frac{g_{i} b_{i}}{\sigma_{u}^{0}-b_{i}}=\frac{g_{i} b_{i}}{\sigma_{M}^{0} t-b_{i}}
\end{gathered}
$$

where

$$
b_{i}=\sigma_{i}-g_{i} s_{i}, \quad \sigma_{i}=\sigma_{u}^{0}\left(s_{i}\right), \quad t_{i}=\frac{\sigma_{i}}{\sigma_{M}^{0}} .
$$

With respect to each interval $\left[s_{i}, s_{i+1}\right]$ the stability equations are in the form similar to (4.1).

Taking the solution

$$
u=\sum_{n=1}^{N_{0}} A_{n} U(t) \cos n \theta, \quad v=\sum_{n=1}^{N_{0}} B_{n} V(t) \sin n \theta, \quad w=\sum_{n=1}^{N_{0}} C_{n} W(t) \cos n \theta .
$$

where $A_{n}, B_{n}, C_{n}$ - constants and substituting them into equations (4.1) we obtain

$$
\begin{aligned}
\Phi(t, \theta) \equiv & \sum_{n=1}^{N_{0}}\left\{A _ { n } \left[\left(4 U^{\prime \prime} t+4 U^{\prime}\right) \Phi^{\prime}-\left(4+\frac{n^{2}}{\sin ^{2} \alpha}\right) \frac{U}{t} \Phi^{\prime}+\right.\right. \\
& \left.+\bar{N}\left(3 U^{\prime \prime} t+3 U^{\prime}-\frac{n^{2}}{\sin ^{2} \alpha} \frac{U}{t}+3 \frac{\partial \bar{N}}{\partial t} \frac{U^{\prime}}{t}\right)\right]+ \\
& +B_{n}\left[\frac{\Phi^{\prime} n}{\sin \alpha}\left(3 V^{\prime}-5 \frac{V}{t}\right)+\bar{N}\left(\frac{n}{\sin \alpha} V^{\prime}-\frac{n^{2}}{\sin \alpha} \frac{V}{t}\right)\right]+ \\
& \left.+C_{n} 2 \Phi^{\prime} \operatorname{cotg} \alpha\left(W^{\prime}+2 \frac{V}{t}\right)\right\} \cos n \theta=0
\end{aligned}
$$




$$
\begin{aligned}
& \Psi(t, \theta) \equiv \sum_{n=1}^{N_{0}}\left\{A_{n}\left[\Phi^{\prime} \frac{n}{\sin \alpha}\left(-3 U^{\prime}-5 \frac{U}{t}\right)+\bar{N} \frac{n}{\sin \alpha}\left(-U^{\prime}-\frac{U}{t}\right)+\frac{n}{\sin \alpha} \frac{U}{t} \frac{\partial \bar{N}}{\partial t}\right]+\right. \\
&+B_{n}\left[\Phi^{\prime}\left(t V^{\prime \prime}+V^{\prime}-\frac{V}{t}\right)-\frac{4 n^{2} \Phi^{\prime}}{\sin ^{2} \alpha} \frac{V}{t}+\bar{N}\left(t V^{\prime \prime}+V^{\prime}-\frac{V}{t}\right)+\frac{\partial \bar{N}}{\partial t}\left(t V^{\prime}-V\right)\right]- \\
&\left.-C_{n} \Phi^{\prime} \frac{4 n}{\sin \alpha} \frac{W}{t} \operatorname{cotg} \alpha\right\} \sin n \theta=0 \\
& \Omega(t, \theta) \equiv \\
& \sum_{n=1}^{N_{0}}\left\{A_{n}\left[24 \Phi^{\prime} \operatorname{cotg} \alpha\left(\frac{l}{h}\right)^{2}\left(U^{\prime}+2 \frac{U}{t}\right)\right]+B_{n}\left[48 \Phi^{\prime} \operatorname{cotg} \alpha \frac{n}{\sin \alpha}\left(\frac{l}{h}\right)^{2} \frac{V}{t}\right]\right. \\
&+ C_{n} \Phi^{\prime}\left[4 W^{(4)} t+8 W^{(3)}-4 \frac{W^{\prime \prime}}{t}-8 \frac{n^{2}}{\sin ^{2} \alpha} \frac{W^{\prime \prime}}{t}+4 \frac{W^{\prime}}{t}+\right. \\
&\left.+\left(4 \frac{n^{2}}{\sin ^{2} \alpha}-16\right) \frac{n^{2}}{\sin ^{2} \alpha} \frac{W}{t^{3}}+48\left(\frac{l}{h}\right)^{2} \operatorname{cotg}^{2} \alpha \frac{W}{t}\right]+ \\
&+C_{n} \bar{N}\left[3 W^{(4)} t+6 W^{(3)}-4 \frac{n^{2}}{\sin ^{2} \alpha} \frac{W^{\prime \prime}}{t}+4 \frac{n^{2}}{\sin ^{2} \alpha} \frac{W^{\prime}}{t}-4 \frac{n^{2}}{\sin ^{2} \alpha} \frac{W}{t^{3}}\right]+ \\
&+C_{n} p 18 t g \alpha\left(\frac{l}{h}\right)^{3}\left[-t^{2} W^{\prime \prime}-2 t W^{\prime}+2 \frac{n^{2}}{\sin ^{2} \alpha}-W\right]+ \\
&+C_{n}\left[6 \frac{\partial \bar{N}}{\partial t} W^{(3)} t+3 \frac{\partial^{2} \bar{N}}{\partial t^{2}} t W^{\prime \prime}\right]+ \\
&\left.+C_{n}\left[6 \frac{\partial \bar{N}}{\partial t} W^{\prime \prime}-4 \frac{n^{2}}{\sin ^{2} \alpha} \frac{\partial \bar{N}}{\partial t} \frac{W^{\prime}}{t}+4 \frac{n^{2}}{\sin ^{2} \alpha} \frac{W}{t^{2}} \frac{\partial \bar{N}}{\partial t}\right]\right\} \cos n \theta=0
\end{aligned}
$$

Functions $U(t), V(t), W(t)$ are chosen, such that the boundary conditions (3.2), (3.3) are satisfied.

$$
U(t)=t(1-t)^{2}, \quad V(t)=t(1-t), \quad W(t)=t^{2}(1-t)^{3} .
$$

Applying the Bubnov - Galerkin's method into equations (4.2), we obtain

$$
\begin{aligned}
& \sum_{j=0} \int_{t_{j}}^{t_{j+1}} d t \int_{0}^{2 \pi} \Phi(t, \theta) t(1-t)^{2} \cos k \theta d \theta=0 \\
& \sum_{j=0} \int_{t_{j}}^{t_{j+1}} d t \int_{0}^{2 \pi} \Psi(t, \theta) t(1-t) \sin k \theta d \theta=0 \\
& \sum_{j=0} \int_{t_{j}}^{t_{j+1}} d t \int_{0}^{2 \pi} \Omega(t, \theta) t^{2}(1-t)^{3} \cos k \theta d \theta=0 .
\end{aligned}
$$

$$
\text { with } k=\overline{0, N_{0}} \text {. }
$$

Using the orthogonality of trigonometric functions we can see that with respect to each $k \equiv n$ obtaining 3 linearly algebraic homogeneous equations of unknown $A_{n}, B_{n}, C_{n}$ separately; totally we get $N_{0}$ sets of 3 separate equations of unknown with $n=1,2, \cdots N_{0}$ 
respectively. Since $A_{n}, B_{n}, C_{n}$ are not simultancously equal to zero, so the determinant of their coefficients must be vanish, it leads to the relationship for determining the critical load.

About the value $t_{s}=x_{s} / l$ which can be defined by using the iterative method, so called elastic solution method. At the first iteration $k=0$ suppose the shell in elastic state, the critical load $p_{c r}^{(0)}$ is determined as in [7], then the value $\sigma_{M}^{(0)}$ is known. If $\sigma_{M}^{(0)} t<\sigma_{s}$ for all $t$, the iterative process can be finished and the instability of shell occurs in elastic range. If $\sigma_{M}^{(0)} t \geqslant \sigma_{s}$, we calculate $t_{s}^{(0)}=\sigma_{s} / \sigma_{M}^{(0)}$ for getting the number of intervals from $t_{s}^{(0)}$ to 1 , on which $\Phi^{\prime}$ takes different constant values and then substitute into (4.3) for the next iteration.

Note that in each iteration one obtains $N_{0}$ values of $p$, the minimum value of which is chosen for the critical $p_{c r}$. Iterative process can be finished when the difference of results in two consecutive iterations is smaller than a given small value. Using just obtained critical load $p_{c r}$ the elasto - plastic zones boundary can be defined as follows

$$
\frac{x_{s}}{l}=t_{s}=\frac{2 \sigma_{s} h}{\sqrt{3} l p_{c r} \operatorname{tg} \alpha} .
$$

\section{NUMERICAL EXAMPLE}

Consider in turn the stability problem of conical shells made of different materials with:

- Linearly hardening characteristics

$$
E=2,6 \cdot 10^{6}\left(\mathrm{kG} / \mathrm{cm}^{2}\right), \quad \sigma_{s}=4 \cdot 10^{3}\left(\mathrm{kG} / \mathrm{cm}^{2}\right), \quad g=0,43 \cdot 10^{6}\left(\mathrm{kG} / \mathrm{cm}^{2}\right) .
$$

- General hardening characteristics

$$
E=2,6.10^{6}\left(\mathrm{kG} / \mathrm{cm}^{2}\right), \quad \sigma_{s}=4.10^{3}\left(\mathrm{kG} / \mathrm{cm}^{2}\right) ;
$$

the relation $\sigma_{u}^{0}=\Phi(s)$ is given in the Table 1 .

Table 1. Relation between stress intensity and hardening modulus

\begin{tabular}{|c|l|l|l|l|l|l|l|l|}
\hline$\sigma_{i}$ & 4000 & 4260 & 4440 & 4540 & 4640 & 4720 & 4780 & 4900 \\
\hline$g_{i} \cdot 10^{6}$ & 0.853 & 0.48 & 0.42 & 0.32 & 0.26 & 0.23 & 0.22 & 0.20 \\
\hline$\sigma_{i}$ & 5000 & 5080 & 5160 & 5400 & 5500 & 5620 & 5700 & 5800 \\
\hline$g_{i} \cdot 10^{6}$ & 0.16 & 0.15 & 0.14 & 0.12 & 0.12 & 0.12 & 0.11 & 0.10 \\
\hline
\end{tabular}

- Elasto - plastic material (almost perfectly plastic material)

$$
E=2.6 \cdot 10^{6}\left(k G / \mathrm{cm}^{2}\right), \quad \sigma_{s}=4.10^{3}\left(k G / \mathrm{cm}^{2}\right), \quad g=0.08 .10^{6}\left(\mathrm{kG} / \mathrm{cm}^{2}\right) .
$$

Firstly, the calculation is carried out with a conical shell of $\alpha=\pi / 4$, the series expansion from $n=1$ to $n=15$ and 10 iterations. The results of critical loads depending on slenderness $l / h$ of the shell with different materials are given in the Table 2 and illustrated on the Fig. 1. 
Table 2. Relation between critical loads and slenderness with various materials

\begin{tabular}{|c|c|c|c|c|c|c|c|c|c|}
\hline \multirow{2}{*}{$\begin{array}{c}p_{c r} \\
\left(k G / \mathrm{cm}^{2}\right)\end{array}$} & \multicolumn{9}{|c|}{$l / h$} \\
\hline & 500 & 600 & 700 & 800 & 900 & 1000 & 1100 & 1200 & 1300 \\
\hline elastic & 25.50 & 17.61 & 12.93 & $9 . \overline{90}$ & 7.78 & 6.32 & 5.21 & 4.38 & 3.72 \\
\hline $\begin{array}{c}\text { linear } \\
\text { hardening }\end{array}$ & 17.07 & 13.70 & 11.05 & 8.50 & 7.37 & 6.32 & 5.21 & 4.38 & 3.72 \\
\hline $\begin{array}{c}\text { general } \\
\text { hardening }\end{array}$ & 13.00 & 10.87 & 8.96 & 7.90 & 7.03 & 6.32 & 5.21 & 4.38 & 3.72 \\
\hline $\begin{array}{l}\text { perfectly } \\
\text { plastic }\end{array}$ & 10.00 & 8.59 & 7.39 & 6.48 & 5.77 & 5.30 & 5.21 & 4.38 & 3.72 \\
\hline
\end{tabular}

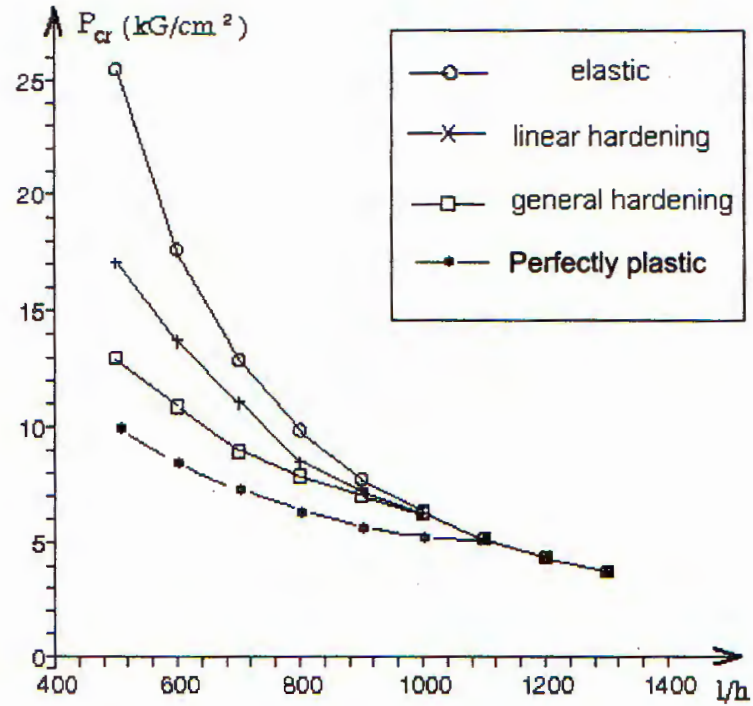

Fig.1. Effect of hardening characteristics on critical loads

Secondly, analysis is fulfilled with a conical shell made of general hardening material, the open angle at the shell top varies from $\pi / 4$ to $\pi / 2$, while the slenderness $l / h=400$. Obtained results are presented in the Table 3.

Table 3. Relation between critical loads, elastic- plastic boundary and open angle

\begin{tabular}{|c|l|l|l|l|l|l|}
\hline$\alpha$ & 0.78 & 0.80 & 0.82 & 0.84 & 0.88 & 0.90 \\
\hline$p_{c r}\left(\mathrm{kG} / \mathrm{cm}^{2}\right)$ & 16.45 & 14.5 & 13.0 & 6.4 & 2.7 & 0.9 \\
\hline$t_{s}=x_{s} / l$ & 0.7 & 0.75 & 0.79 & 1.0 & 1.0 & 1.0 \\
\hline
\end{tabular}

\section{DISCUSSION}

From the results we can see that

- For conical shells when the ratio $l / h$ increases, then the critical load decreases while the value $x_{s} / l$ increases, i.e. the plastic zone is reduced. The ratio $l / h$ is reaching some value the loss of stability of the shell will occur in elastic range (in considered example with the shell of $\alpha=\pi / 4$ and $l / h \geqslant 1000$ ). 
- With materials of the same $E, \sigma_{s}$ and the shell of the same ratio $l / h$ and open angle $\alpha$ the critical loads decrease in turn elastic, linear hardening, general hardening and perfectly plastic material. The greater hardening modulus $\Phi^{\prime}(s)$ of material results in greater critical load.

- With the same material and the same ratio $l / h$ the critical loads decrease when the open angle $\alpha$ at shell top increases, i.e for a flatter conical shell the critical load is smaller.

In conclusion, an analysis procedure has been developed for solving the elastoplastic stability problem of conical shells.

Acknowledgement. The present publication is supported by the National Council for Natural Sciences.

\section{REFERENCES}

1. J. Lubliner, Plasticity Theory, Macmillan publishing company, 1990.

2. Hill R, Plastic deformation and instability in thin- walled tubes under combined loading: a general theory, Journal of Mech. Phys of Solids 47 (1999) 921 - 933.

3. Dao Huy Bich, Theory of Elastoplastic Processes, VNU Publishing House, 1999.

4. Ulo Lepik, Bifurcation analysis of elastic plastic cylindrical shells, Int. Journal of Non - linear Mechanics 34 (1999) 299 - 311.

5. Dao Huy Bich, On the elasto-plastic stability problem of shells of revolution, Vietnam Journal of Mechanics 25 (1) (2003) 9 - 18.

6. Dao Huy Bich, Tran Thanh Tuan, Vu Khac Bay, On the elasto-plastic stability problem of conical shells, Proc. of 7-th Nat. Conf. on Solid Mechanics 2004, pp. 22 30.

7. D. O. Brush, B. O. Almorth, Buckling of Bars, Plates and Shells, Mc Graw - Hill, 1975.

Received November 17, 2005

\section{ÁNH HƯờNG CƯA DẶC TRƯNG TÁI BỀN CƯA VẠT LIỆ ĐẾN TẢI TRỌNG TớI HẠN TRONG BÀI TOÁN ỔN ĐỊNH ĐÀN-DE்O CƯA VỎ NÓN}

Trong bài báo này đã thiết lập các phương trình cơ bản của bài toán ổn định ngoài giới hạn đàn hồi của vó nón dựa trên lý thuyết quá trình đàn déo và tiêu chuẩn tồn tại các dạng cân bằng lân cận, khi trạng thái màng trước khi mất ổn định là không thuần nhất. Lời giải của bài toán nhận được nhờ áp dụng phương pháp Bubnov-Galerkin và phương pháp tham số tải, qua đó khăo sát ảnh hường của đặc trưng tái bền của vật liệu đến tải trọng tới hạn của vỏ nón chịu tác dụng của áp suất ngoài. 